JOURNAL OF

FUNCTION SPACES AND APPLICATIONS

Volume 4, Number 1 (2006), 85-90
(C) 2006, Scientific Horizon http://www.jfsa.net

\title{
New proof of Nagnibida's theorem
}

\author{
Mubariz T. Karaev \\ (Communicated by Nicolae Popa)
}

2000 Mathematics Subject Classification. Primary 47B38.

Keywords and phrases. Duhamel product, holomorphic function, closed ideal, integration operator.

\footnotetext{
Abstract. Using the Duhamel product for holomorphic functions we give a new proof of Nagnibida's theorem on unicellularity of integration operator $J_{\alpha},\left(J_{\alpha} f\right)(z)=\int_{\alpha}^{z} f(t) d t$, acting in the space $\mathcal{H} o \ell(\Omega)$.
}

Let $\alpha$ be a fixed complex number, $\Omega$ be a simple-connected domain in the plane containing the number $\alpha$, and assume that $\Omega$ is star-shaped with respect to the $\alpha$ in the sense that $z \in \Omega$ implies $\lambda z+(1-\lambda) \alpha \in \Omega$ for each $\lambda \in(0,1)$. Let $\mathcal{H} o \ell(\Omega)$ be the vector space of all functions holomorphic in $\Omega$ with the topology of compact convergence, and let $J_{\alpha}$ denotes the integration operator acting in $\mathcal{H} o \ell(\Omega)$ by formula

$$
\left(J_{\alpha} f\right)(z)=\int_{\alpha}^{z} f(t) d t
$$

where integral is taken on the line segment with the ends $\alpha$ and $z$. 
We denote by $I^{p}$ the set of functions with a zero at the point $\alpha$ of order greater than or equal $p$, i.e.,

$$
I^{p} \stackrel{\text { def }}{=}\left\{f \in \mathcal{H} o \ell(\Omega): f(\alpha)=f^{\prime}(\alpha)=\ldots=f^{(p-1)}(\alpha)=0\right\}
$$

It is clear that all the subspaces $I^{p}, p \geq 1$, are closed $J_{\alpha}$-invariant subspaces (i.e., $I^{p} \in \operatorname{Lat}\left(J_{\alpha}\right), p \geq 1$ ) and if $p>q$, then $I^{p} \subset I^{q}$. The operator $J_{\alpha}$ has been extensively studied by Nagnibida [?].

In this short communication we give a new proof of the following theorem of Nagnibida [3, Theorem 5] on unicellularity of the operator $J_{\alpha}$ in the space $\mathcal{H} o \ell(\Omega)$. We recall that an operator $A$ acting in the topological vector space $X$ is called unicellular if its lattice of invariant subspaces Lat $(A)$ is linearly ordered with respect to inclusion.

Theorem. Lat $\left(J_{\alpha}\right)=\left\{I^{p}: p \geq 1\right\}$, i.e., the operator $J_{\alpha}$ is unicellular in the space $\mathcal{H o l}(\Omega)$.

Proof. In the proof of theorem we shall essentially use the similar arguments of the paper [?]. For every $f, g \in \mathcal{H} o \ell(\Omega)$ let us define the following product:

$$
\begin{aligned}
(f \underset{\alpha}{\circledast} g)(z) & \stackrel{\text { def }}{=} \frac{d}{d z} \int_{\alpha}^{z} f(z+\alpha-t) g(t) d t \\
& =\int_{\alpha}^{z} f^{\prime}(z+\alpha-t) g(t) d t+f(\alpha) g(z),
\end{aligned}
$$

where integral is taken on the line segment with the ends $\alpha$ and $z$. (For $\alpha=0$ this is the so-called Duhamel product, see [?]). Clearly $f \underset{\alpha}{\circledast} g$ also belongs to $\mathcal{H} o \ell(\Omega)$, and with this multiplication $\mathcal{H} \circ \ell(\Omega)$ becomes an algebra. One can use results of operational calculus [?], [?] and analytic continuation to show that $\mathcal{H o \ell}(\Omega)$ is commutative and associative(it is actually clear from (1) that $f(z) \equiv 1$ is the unit for $\mathcal{H o \ell}(\Omega)$, and a simple change of variables shows commutativity). It is clear from (1) that $J_{\alpha} f(z)=(z-\alpha) \circledast f(z)$. Then by considering this and the fact that the system $\left\{(z-\alpha)^{n}\right\}_{n=0}^{\infty}$ is complete in $\mathcal{H o \ell}(\Omega)$ (which is obtained from the well-known completeness criterion of Banach in the Frechet spaces and classical Runge's approximation theorem), it is not difficult to show that the problem of description of all closed $J_{\alpha}$-invariant subspaces is equivalent to the description of all closed ideals of the algebra $\mathcal{H o \ell}(\Omega)$ with multiplication as $\circledast$. We shall now describe the all closed ideals of the 
algebra $(\mathcal{H} o \ell(\Omega), \underset{\alpha}{\circledast})$ by showing that any nontrivial closed ideals has the form $I^{p}$ for some $p \geq 1$. The proof will be done in several steps:

Step 1. Let $f \in(\mathcal{H} o \ell(\Omega), \underset{\alpha}{\circledast})$. Then $f$ is $\underset{\alpha}{\circledast}$ - invertible if and only if $f(\alpha) \neq 0$.

Proof. Since $(f \underset{\alpha}{\circledast} g)(\alpha)=f(\alpha) g(\alpha)$, it is clear that if $f$ is $\circledast$ - invertible, then $f(\alpha) \neq 0$. We now prove that if $f(\alpha) \neq 0$ then $f$ is $\circledast \alpha$-invertible. Without loss of generality, we assume that $f(\alpha)=1$.

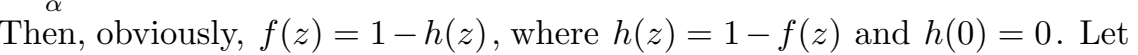
$\Omega^{\prime}$ be an arbitrary bounded domain, star-shaped with respect to the point $\alpha$, and such that its closure is contained in $\Omega$. Choose the number $M>0$ such that $\left|h^{\prime}(z)\right| \leq M$ for $z \in \Omega^{\prime}$. Then

$$
|h(z)|=\left|\int_{\alpha}^{z} h^{\prime}(t) d t\right| \leq M r_{\alpha}
$$

for $z \in \Omega^{\prime},|z-\alpha|=r_{\alpha}$. Let $h^{[n]}$ denote the $\underset{\alpha}{\circledast}-$ product of $h$ with itself $n$ times for $n \geq 0$, where $h^{[0]}(z) \equiv 1$. We shall prove by induction that

$$
\left|h^{[n]}(z)\right| \leq \frac{M^{n} r_{\alpha}^{n}}{n !}
$$

and

$$
\left|\frac{d}{d z} h^{[n]}(z)\right| \leq \frac{M^{n} r_{\alpha}^{n-1}}{(n-1) !}
$$

for $z \in \Omega^{\prime}$. Indeed, for this purpose, assume that

$$
\left|h^{[k]}(z)\right| \leq \frac{M^{k} r_{\alpha}^{k}}{k !}
$$

and

$$
\left|\frac{d}{d z} h^{[k]}(z)\right| \leq \frac{M^{k} r_{\alpha}^{k-1}}{(k-1) !}
$$

for $z \in \Omega^{\prime}$. Then, by considering that $h(\alpha)=0$, we obtain that

$$
\left|h^{[k+1]}(z)\right|=\left|\frac{d}{d z} \int_{\alpha}^{z} h(z+\alpha-t) h^{[k]}(t) d t\right|
$$




$$
\begin{aligned}
& =\left|\int_{\alpha}^{z} h^{\prime}(z+\alpha-t) h^{[k]}(t) d t\right| \\
& \leq \int_{\alpha}^{z}\left|h^{\prime}(z+\alpha-t)\right|\left|h^{[k]}(t)\right||d t| \\
& \leq \frac{M^{k+1}}{k !} \int_{\alpha}^{z}|t-\alpha|^{k}|d(t-\alpha)| \\
& =\frac{M^{k+1}}{k !} \int_{0}^{r_{\alpha}} \rho_{\alpha}^{k} d \rho_{\alpha} \\
& =\frac{M^{k+1} r_{\alpha}^{k+1}}{(k+1) !}
\end{aligned}
$$

and

$$
\begin{aligned}
\left|\frac{d}{d z} h_{\alpha}^{[k+1]}(z)\right| & =\left|\int_{\alpha}^{z} h^{\prime \prime}(z+\alpha-t) h^{[k]}(t) d t+h^{\prime}(\alpha) h^{[k]}(z)\right| \\
& =\left|\int_{\alpha}^{z} h^{\prime}(z+\alpha-t) \frac{d}{d t} h^{[k]}(t) d t\right| \\
& \leq \frac{M^{k+1}}{(k-1) !} \int_{0}^{r_{\alpha}} \rho_{\alpha}^{k-1} d \rho_{\alpha} \\
& =\frac{M^{k+1} r_{\alpha}^{k}}{k !}
\end{aligned}
$$

Thus the series $g(z)=\sum_{n=0}^{\infty} h^{[n]}(z)$ and $\sum_{n=0}^{\infty} \frac{d}{d z} h^{[n]}(z)$ are majorized respectively by the series

$$
\sum_{n=0}^{\infty} \frac{M^{n} r_{\alpha}^{n}}{n !}=e^{M r_{\alpha}} \leq e^{M d}
$$

and

$$
M \sum_{n=1}^{\infty} \frac{M^{n-1} r_{\alpha}^{n-1}}{(n-1) !}=M e^{M r_{\alpha}} \leq M e^{M d},
$$

where $d$ is the diameter of $\Omega^{\prime}$. Consequently, $g$ is holomorphic in $\Omega^{\prime}$ and because of the arbitrariness of $\Omega^{\prime}, g$ is holomorphic in $\Omega$. Finally $f \underset{\alpha}{\circledast} g=(1-h) \underset{\alpha}{\circledast} g=(1-h) \underset{\alpha}{\circledast} \sum_{n=0}^{\infty} h^{[n]}=\mathbf{1}$.

Step 2. Let $f, g \in \mathcal{H} o \ell(\Omega)$. Suppose $f$ has a zero at the point $\alpha$ of order $p, g$ has a zero of order $q$, and $p \leq q$. Then there exists $h \in \mathcal{H} o \ell(\Omega)$ with a zero of order $q-p$ such that $f \circledast h=g$. 
Proof. Since $f^{(p)} \in \mathcal{H} o \ell(\Omega)$ and $f^{(p)}(\alpha) \neq 0$, by Step 1 there exists

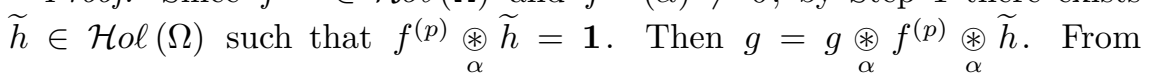
(1) and integration by parts $p$ times we obtain $g \underset{\alpha}{\circledast} f^{(p)}=g^{(p)} \underset{\alpha}{\circledast} f$. Thus

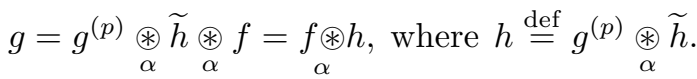

Step 3. $(\mathcal{H} o \ell(\Omega), \underset{\alpha}{\circledast})$ is a local ring and any nontrivial closed ideal in $(\mathcal{H} o \ell(\Omega), \underset{\alpha}{\circledast})$ has the form $I^{p}$ for some $p \geq 1$.

Proof. If $f \in(\mathcal{H} o \ell(\Omega), \underset{\alpha}{\circledast})$ and $f(\alpha)=0$, then it follows from $(1)$ that $(f \underset{\alpha}{\circledast} g)(\alpha)=0$ for $g \in(\mathcal{H} o \ell(\Omega), \underset{\alpha}{\circledast})$. Thus $I^{1}$ form an ideal. From the Step 1 this ideal must be maximal and is only maximal ideal of $(\mathcal{H} o \ell(\Omega), \underset{\alpha}{\circledast})$. Thus $(\mathcal{H} o \ell(\Omega), \underset{\alpha}{\circledast})$ is local ring. Each $I^{p}$ is an ideal (closed) of $(\mathcal{H o \ell}(\Omega), \underset{\alpha}{\circledast})$ as can be seen from (1). We now prove that other nontrivial ideals not exist. Indeed, if $I$ is any nontrivial ideal in $(\mathcal{H} o \ell(\Omega), \underset{\alpha}{\circledast})$, then $I$ has an element $f$ of lowest order $p$. By Step 2, if $g \in I^{p}$, then there exists $h \in \mathcal{H} o \ell(\Omega)$ such that $g=h \circledast f$, and hence $I^{p} \subseteq I$. If $I^{p} \neq I$, then $I$ has an element of order less than or equal to $p-1$, a contradiction. Thus $I=I^{p}$. Consequently, the only ideals of $(\mathcal{H} o \ell(\Omega), \underset{\alpha}{\circledast})$ are the $I^{p}, p \geq 1$.

In addition we observe that the sequence

$$
(\mathcal{H} o \ell(\Omega), \underset{\alpha}{\circledast})=I^{0} \supset I^{1} \supset I^{2} \supset \ldots \supset\{0\}
$$

is a filtration of $(\mathcal{H} o \ell(\Omega), \underset{\alpha}{\circledast})$ and that $I^{p} / I^{p+1}$ is isomorphic to the complex numbers.

Thus, by Step 3 the proof of theorem is established.

Remark. Note that the invertibility criterion with respect to the Duhamel product $\circledast$ can also be easily deduced from the corresponding result of Wigley $[4$, Theorem] for $\alpha=0$. Indeed, there is a canonical algebra isomorphism $\Gamma$ between the spaces $\mathcal{H o} \ell(\Omega)$ and $\mathcal{H} o \ell\left(\Omega_{0}\right)$ where $\Omega_{0}=\Omega-\alpha$, when endowed with their corresponding Duhamel products. 
The map is obviously defined by $\Gamma f(z)=f(z+\alpha)$. Besides, $\Gamma$ implements a similarity between the corresponding integration operators, so from the invariant subspace point of view, it is enough to assume that $\alpha=0$.

Acknowledgement. I am grateful to the referee for his useful suggestions.

\section{References}

[1] G. Krabbe, Operational Calculus, New York, Springer-Verlag, 1970.

[2] J. Mikusinski, Operational Calculus, 1956.

[3] N. I. Nagnibida, Description of commutants of integration operator in analytic spaces, Siberian Math.J., 22 (1982), 748-752; translation from Sib. Mat. Zh., 22 (1981), 127-131.

[4] N. M. Wigley, The Duhamel product of analytic functions, Duke Math. J., 41 (1974), 211-217.

Suleyman Demirel University

Department of Mathematics

32260 Isparta

Turkey

(E-mail : garayev@fef.sdu.edu.tr)

(Received: March 2005) 


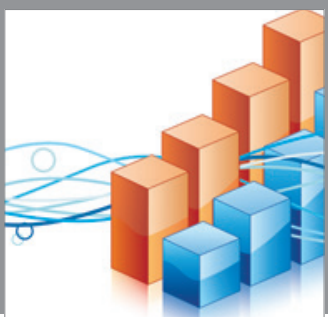

Advances in

Operations Research

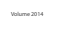

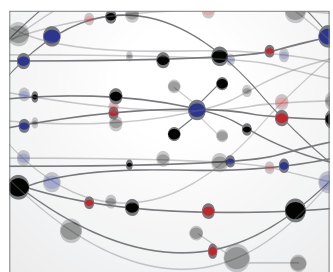

\section{The Scientific} World Journal
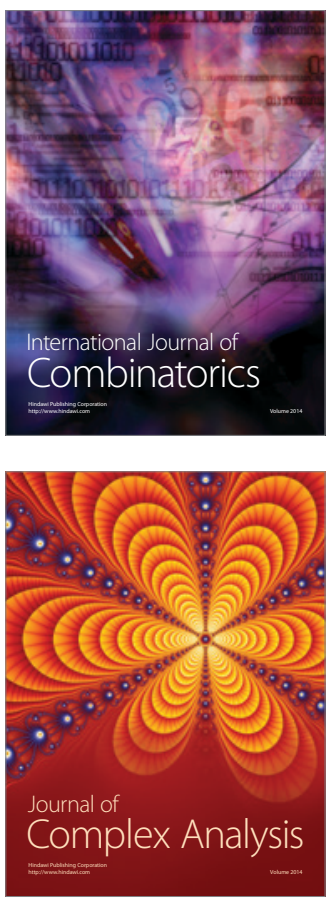

International Journal of

Mathematics and

Mathematical

Sciences
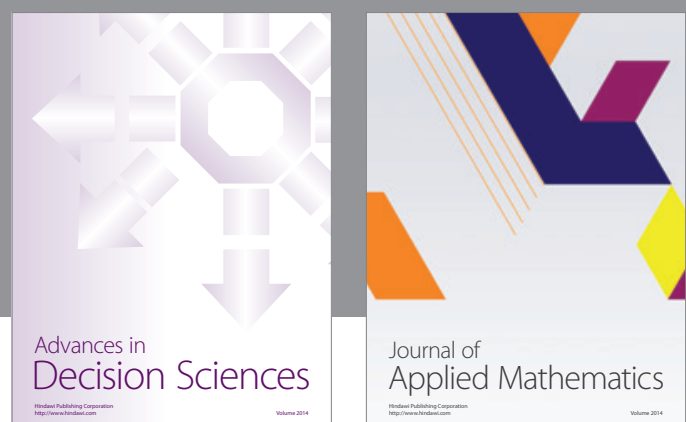

Journal of

Applied Mathematics
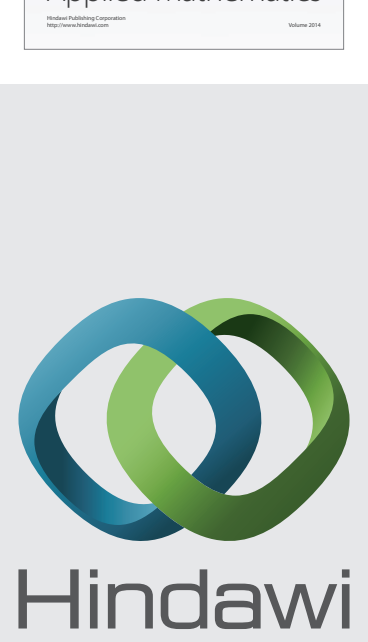

Submit your manuscripts at http://www.hindawi.com
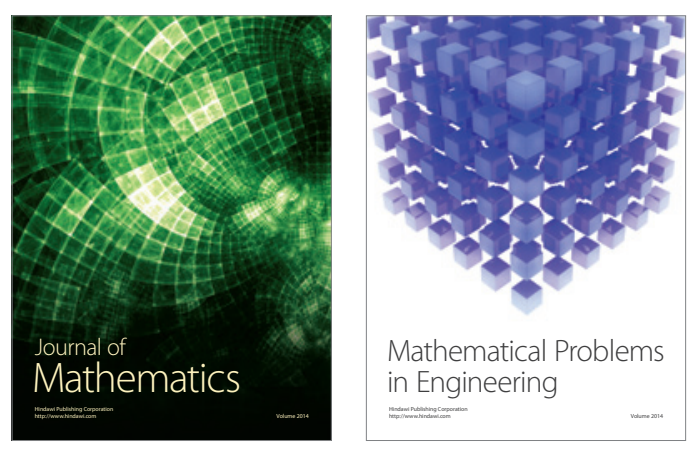

Mathematical Problems in Engineering
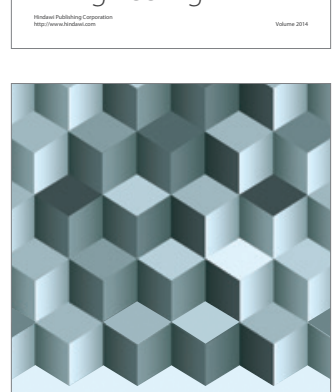

Journal of

Function Spaces
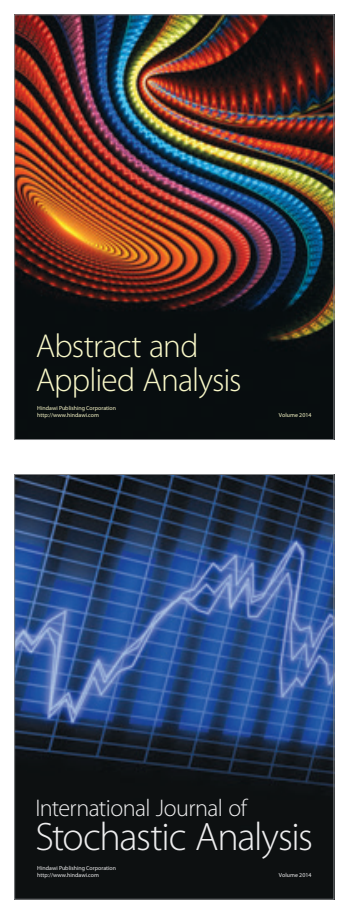

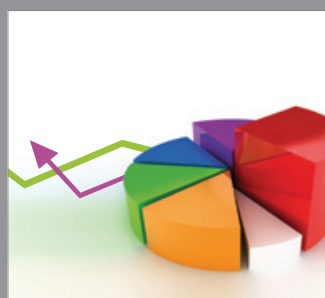

ournal of

Probability and Statistics

Promensencen
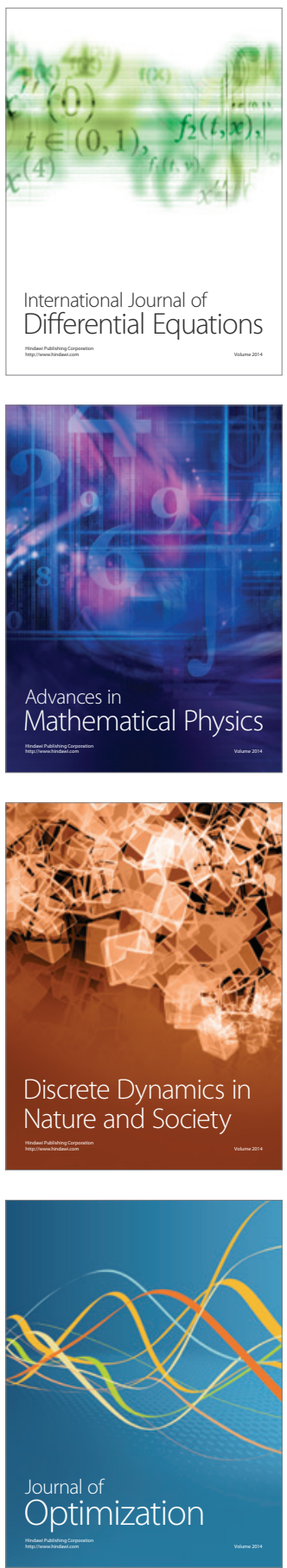